\title{
Inelastic Diffraction at Heavy Ion Colliders
}

\author{
Sebastian N. White ${ }^{\mathrm{a}}$ \\ ${ }^{a}$ Brookhaven National Laboratory, \\ Upton, N.Y. 11973, USA
}

The heavy ion physics approach to global event characterization has led us to instrument the forward region in the PHENIX experiment at RHIC. In heavy ion collisions this coverage yields a measurement of the "spectator" energy and its distribution about the beam direction. This energy flow is the basis of event-by-event determination of the centrality and reaction plane which are key to analyzing particle production in heavy ion collisions. These same tools have also enabled a unique set of measurements on inelastic diffraction with proton, deuteron and gold ion beams in the PHENIX experiment. We present first new results on this topic and discuss briefly the opportunity for diffractive physics with Heavy Ion beams at the LHC.

\section{Introduction}

Like the LHC, the RHIC collider is a dual function machine providing collisions of protons as well as heavy ions. Unlike the LHC, the experiments at RHIC have been designed completely with the goals of heavy ion physics in mind and then subsequently used also for measurements with (polarized) proton colliding beams. In many cases the choice of parameters in detector design have had interesting consequences for the types of measurement that can be carried out with pp collisions but nowhere is this so clear as in the case of forward instrumentation.

\subsection{Heavy Ion Goals}

The primary goals of forward instrumentation at RHIC are

- to measure absolute luminosity

- to provide a truly minimum bias trigger

- to characterize the initial conditions of individual events(reaction plane orientation, impact parameter) outside the mid-rapidity region

The interaction region geometry, depicted in Fig. 1 , provides a location at $\mathrm{z}= \pm 18 \mathrm{~m}$ of maximum momentum dispersion where "spectator fragments" from the colliding nuclei are resolved primarily according to their charge-to-mass ratio (Z/A). An intermediate impact parameter event is shown in Fig. 2. RHIC experiments were designed to characterize the reaction plane and impact parameter of individual events. The number of forward going "spectator nucleons" leaving the collision is anti-correlated with the number of participants:

$N_{\text {spectator }}+N_{\text {participant }}=2 \times A$.

In neutron rich ion species stored at RHIC $(\mathrm{Au})$ and $\mathrm{LHC}(\mathrm{Pb})$ most of the information is carried by neutrons which are detected in the RHIC Zero Degree Calorimeters (ZDC's) 2] that were installed in each experiment. Protons are also measured in the PHENIX experiment using a large area hadron calorimeter recycled from AGS experiment E865.

The centrality measurement by spectators is partially diluted by a significant component of recombination/coalescence of the spectator nucleons (ie $n+p \rightarrow \mathrm{d}$ ) which are neither detected in the ZDC or the FCAL. Even for central collisions $\sim 1 / 2$ of the protons coalesce to higher mass fragments [4. So in PHENIX the participant number is sampled by the multiplicity in the $3 \leq|\eta| \leq 4$ region and combined with ZDC energy to form the centrality algorithm.

Early studies of RHIC collisions [3] showed that spectator neutrons are detected in the ZDC 

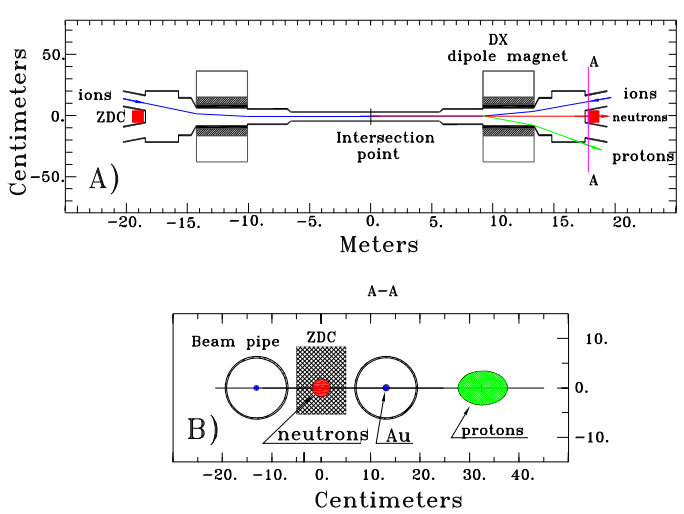

Figure 1. RHIC interaction region layout.

with eff ZDC $\geq 99.5 \%$ in nuclear interactions at all centralities. Even in UltraPeripheral Collisions (UPC) where $R_{\text {impact }} \geq 2 \times R_{\mathrm{Au}}$, beam energy neutrons are evaporated (and detected in the ZDC's) due to the intense electromagnetic interactions between high-Z nuclei [1]. The ZDC is therefore the ideal minimum biased event trigger for nuclear interaction physics. For UPC, the ZDC trigger ("tagged" UPC events-see below) is used to form "rapidity gap" triggers and is the only practical way to access this physics.

Because the dominant UPC process -mutual coulomb dissociation - is calculable with an accuracy of $\sim 5 \%[1$ and cleanly measured [3], it is used as the basis for all absolute luminosity measurements in RHIC.

The reaction plane orientation is best measured in the ZDC using a position sensitive scintillator array at a depth of $2 \times \lambda_{\text {Int }}$ in the calorimeter because of the large directed flow, $v_{1}$, among nucleons at this rapidity [5]. Since the orientation is measured independently in both ZDC arms(left and right) the resolution can be directly derived and we find

$$
\begin{aligned}
0.3 & =\left\langle\cos \left(\psi_{\text {meas }}-\psi_{\text {true }}\right)\right\rangle \\
& =\sqrt{2} \cdot\left\langle\cos \left(\psi_{\text {right }}-\psi_{\text {left }}\right)\right\rangle
\end{aligned}
$$

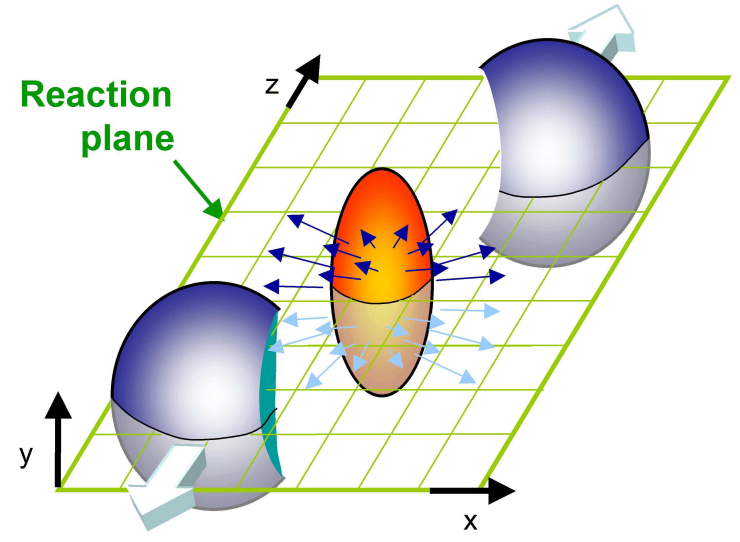

Figure 2. Collision geometry in heavy ion collisions. Both magnitude and direction of the impact parameter are measured by forward "spectator" remnants.

\section{EM Interactions of Heavy Ions}

Ultraperipheral collisions become an important aspect of heavy ion physics at the large c.m. energies available at colliders. At the LHC, with $\mathrm{Pb}+\mathrm{Pb}$ collisions at $\sqrt{S_{N N}}=5.5 \mathrm{TeV}$, processes can be studied that are equivalent to $100 \mathrm{TeV}$ quasi-real photon collisions with a stationary nuclear target [6] opening up a wide range of opportunities [7.

The principal method for analyzing such collisions, the method of equivalent quanta, originated with Fermi 8 who showed that interactions of electrons and $\alpha$-particles with atoms can be reduced to a convolution of an equivalent photon spectra and the known $\gamma-\mathrm{A}$ interaction cross sections. The method was extended to the extreme relativistic case by Weizsacker and Williams and has found many applications in high energy and nuclear physics.

At ion colliders the characteristic strength of interaction $\sim Z_{1} \times Z_{2} \times \alpha$ is large so that multiple photon exchanges lead to the phenomenon of tagging. Additional photon exchanges can cause photo-nuclear breakup of one nucleus which emits a soft neutron 9] detected in the ZDC. Origi- 


\begin{tabular}{|l|c|c|}
\hline Cross Section & STAR $(\mathrm{mb})$ & ref. $(\mathrm{mb})$ \\
\hline$\sigma_{\mathrm{xn}, \mathrm{xn}}^{\rho}$ & $28.3 \pm 2.0 \pm 6.3$ & 27 \\
$\sigma_{1 \mathrm{n}, 1 \mathrm{n}}^{\rho}$ & $2.8 \pm 0.5 \pm 0.7$ & 2.6 \\
\hline$\sigma_{x n, x n}^{\rho \text { (inc.overlap) }}$ & $39.7 \pm 2.8 \pm 9.7$ & - \\
$\sigma_{\mathrm{xn}, 0 \mathrm{n}}^{\rho}$ & $95 \pm 60 \pm 25$ & - \\
$\sigma_{0 \mathrm{n}, 0 \mathrm{n}}^{\rho}$ & $370 \pm 170 \pm 80$ & - \\
\hline$\sigma_{\text {total }}^{\rho}$ & $460 \pm 220 \pm 110$ & 350 \\
\hline
\end{tabular}

Table 1

$\rho$ photoproduction results reproduced from Ref. [10].

nally the tagging requirement was thought of as a penalty factor required to access this physics with a clean experimental trigger. However, as shown in Ref. 9] the tagging fraction is correlated with impact parameter and strongly biases the tagged photon distribution toward higher equivalent energies. We now calculate tagged and untagged photon spectra separately, using this nomenclature below.

\subsection{Diffractive vector meson production at RHIC}

During the $\sqrt{S_{N N}}=130 \mathrm{GeV}$ initial $\mathrm{Au}+\mathrm{Au}$ run, STAR recorded data with a two-prong track requirement (consistent with diffractive $\rho \rightarrow$ $\pi^{+} \pi^{-}$decays) as well as a minibias ZDC trigger 10. The two data samples were examined for diffractive $\rho$ production as well as low mass $\gamma \gamma \rightarrow e^{+} e^{-}$candidates.

The results are summarized in Table. 1, reproduced from Ref. [10] where results are also compared with a calculation of the $\rho$ production cross sections for both the tagged and untagged cases 11]. The cross sections (subscripts 0n and Xn denote 0 and 1-or more neutron tags, respectively) are in reasonable agreement with the calculation. Note that the experimental errors are smallest in the tagged sample.

During the most recent (2004) $\mathrm{Au}+\mathrm{Au}$ run, PHENIX implemented a diffractive trigger sensitive to high mass $e^{+} e^{-}$pairs using a ZDC tag and vetoing on events with activity in the $3 \leq|\eta| \leq 4$ (=BBC) region. The PHENIX experiment has excellent identification capabilities for high mo- mentum electrons due to the highly segmented $(\Delta \eta \times \Delta \Phi=0.01 \times 0.01)$ Electromagnetic $(\mathrm{EM})$ Calorimeter and Ring Imaging Cerenkov counters. The diffractive $e^{+} e^{-}$trigger also required at least one cluster of calorimeter energy with greater than $0.8 \mathrm{GeV}$. The full trigger is

$\mathrm{UPC}=\mathrm{ZDC}($ or $) \cdot \mathrm{BBC}($ not and $) \cdot E M$.

This loose rapidity gap trigger (ie a leading neutron and no activity in at least one BBC arm) is very effective in heavy ion collisions and had a rate of $\leq 0.5 \%$ of the min bias rate. A total of $8.5 \mathrm{M}$ UPC triggers were recorded. Electron candidates were reconstructed requiring consistent EM calorimeter energy and magnetic spectrometer momentum as well as a Cerenkov match. The resultant $e^{+} e^{-}$invariant mass spectrum [12] is shown in Fig. 3. All candidates have a net $p_{t}$ of $\leq 100 \mathrm{MeV}$, consistent with diffractive photoproduction and the expected $\mathrm{Au}$ nuclear form factor. This should be compared to the $\overline{p_{t}} \geq 1$ $\mathrm{GeV} / \mathrm{c}$ we find in non-diffractive $p+p \rightarrow J / \psi+\mathrm{X}$ data .

It should be noted that this spectrum is highly preliminary since, at the time of this initial pass through the data, there has been very little prior experience in PHENIX reconstructing events with such low multiplicities (ie where the interaction vertex is not well constrained). Our calculation of the expected $\mathrm{J} / \psi$ and $e^{+} e^{-}$continuum yield is higher than observed by about a factor of 2. Nevertheless, even at this very early stage in the analysis, it is clear that a diffractive signal of high mass pairs has been seen and the capability for small cross section diffractive physics has been demonstrated.

\section{3. $\mathbf{A u}+\mathbf{d} \rightarrow \mathbf{A u}+n+p$}

Immediately following the first high luminosity $\mathrm{Au}+\mathrm{Au}$ run $\mathrm{RHIC}$ operated as a $\mathrm{d}+\mathrm{Au}$ collider (at $\sqrt{S_{N N}}=200 \mathrm{GeV}$ ). The primary purpose of this run was to directly measure the contribution of initial state nuclear effects on inclusive particle spectra observed in $\mathrm{d}-\mathrm{Au} \rightarrow \pi^{0}+\mathrm{X}$ for example. In order to measure inclusive particle cross sections, it is critical to calibrate the d-Au luminosity. Also, since particle spectra are compared for 


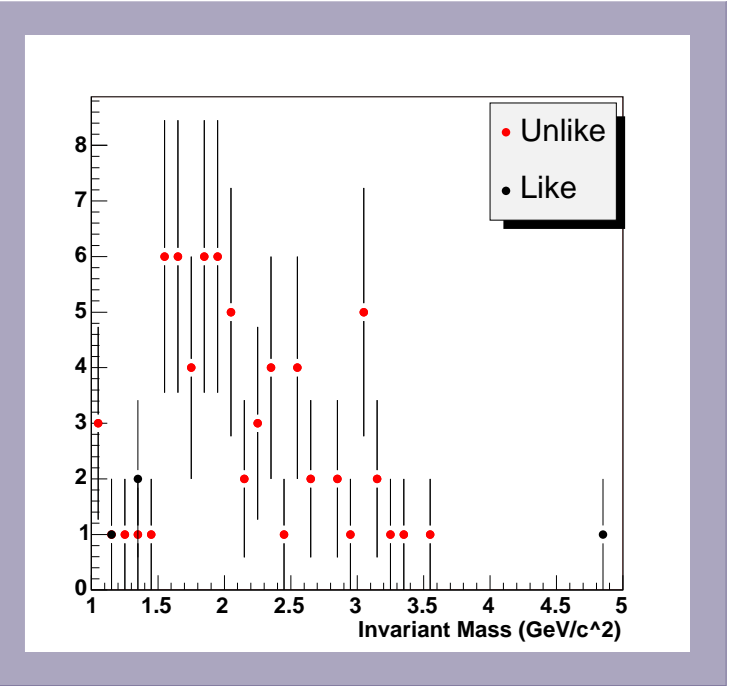

Figure 3. Preliminary PHENIX diffractive $e^{+} e^{-}$ mass spectrum.

an equivalent number of binary collisions, derived from a Glauber model calculation, it is useful to validate this calculation by confirming the corresponding minimum bias cross section.

During the $\mathrm{d}+\mathrm{Au}$ run STAR and PHENIX ran with different min.bias triggers:

$$
\begin{aligned}
\min \cdot \operatorname{bias}(\mathrm{STAR}) & =\mathrm{ZDC}_{\mathrm{Au}-\text { beam }}(\geq 15 \mathrm{GeV}) \\
\text { min.bias }(\mathrm{PHENIX}) & =\mathrm{BBC}_{\text {left }} \cdot \mathrm{BBC}_{\text {right }}
\end{aligned}
$$

which, as pointed out in Ref. 14, have different acceptances for $N N$ collisions with a rapidity gap and hence different cross sections.

In PHENIX we have measured directly the inelastic $\mathrm{d}+\mathrm{Au}$ cross section by normalizing to the theoretical calculation 13] for deuteron photodissociation of $\sigma_{\text {diss }}=1.38 \pm 0.07 \mathrm{~b}$. PHENIX recorded $12 \mathrm{M}$ events with three different triggers enabled: the 2 inelastic triggers of eqn. 4 in "or" with a trigger requiring $\mathrm{ZDC}_{\mathrm{d} \text {-beam }} \geq 15 \mathrm{GeV}$. This last trigger is $100 \%$ efficient for deuteron breakup events since, with a reasonable deuteron input wavefunction, all neutrons fall within the ZDC aperture. Dissociation events have a particularly clean signature in the PHENIX forward detectors since, in the d beam direction, there is an easily resolved $100 \mathrm{GeV}$ energy peak simultaneously in both the ZDC and the proton calorimeter. Also there is no activity in the central detector or the Au beam ZDC.

Ref. 14] predicts a factor of 0.83 suppression of the min.bias(PHENIX) cross section relative to the naive Glauber value of $\sigma_{\text {Glauber }}=2.33 \mathrm{~b}$. Instead we measure, applying a small correction for BBC efficiency:

$\sigma_{\text {PHENIX }(\text { min.bias })}=2.39 \pm 0.24 \mathrm{~b}$,

which differs by $\sim 2$ st.dev. from Ref. [14]. The error on this preliminary result will probably reach $\leq 5 \%$ after further study.

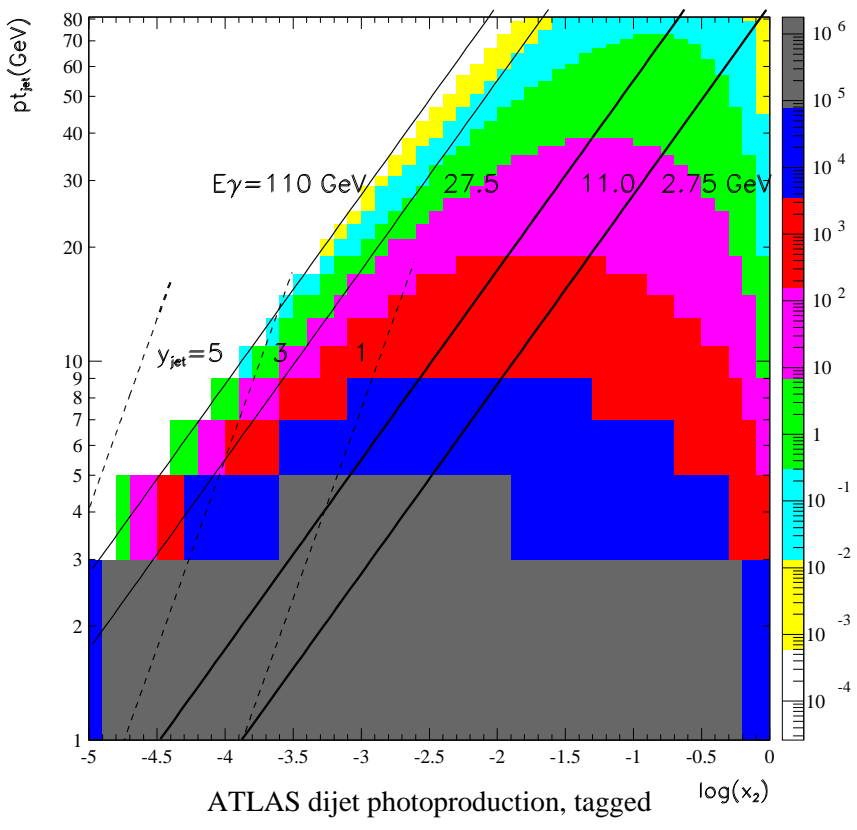

Figure 4. Rates of photo-produced jet pairs from a 1 month run in ATLAS.

4. $p p \rightarrow n+X$

Large $x_{F}$ inclusive cross sections are relevant for analysis of super-GZK cosmic ray candidates and also for LHC luminometry, where this process will be used for beam diagnostics. At RHIC it has a special role in polarimetry since there is 
a large $(\sim 10 \%)$ left-right asymmetry of the neutron angular distribution relative to the up-down polarization of the incident proton. Also a large $(\sim 1 \%) p p \rightarrow n n+\mathrm{X}$ signal is observed which is not reproduced by most event generators (ie DPMJETIII). This signal is the basis of all RHIC $p p$ luminosity monitoring. Further study of these forward physics processes is now under way.

\section{Photoproduction with Heavy Ions at the LHC}

The ATLAS experiment is now constructing ZDC's to be installed in the TAN absorber at $\pm 140 \mathrm{~m}$ from the IP1 interaction region. Full geant simulation of this detector with $\sqrt{S_{N N}}=$ 5.5 TeV $\mathrm{Pb}+\mathrm{Pb}$ collisions has demonstrated a similar energy resolution to that observed at RHIC.

The ATLAS ZDC's will be the primary trigger for diffractive physics and will be combined with low- $p_{t}$ jet or secondary vertex triggers. A likely area of research is the study of photo-produced dijets, $\gamma$-jet pairs and heavy quarks $(Q \bar{Q})$ and also the corresponding diffractive photo-production processes (which have a rapidity gap in both beam directions). We have carried out [15] a calculation of rates for the above processes and the jet-jet case is presented in Fig. 4 for a 1 month run at nominal $\mathrm{LHC} \mathrm{Pb}-\mathrm{Pb}$ luminosities. Even with a jet threshold of $\geq 10-15 \mathrm{GeV}$ the total event rate exceeds $\sim 10^{5}$. We are now studying the ATLAS capability for accessing low $p_{t}$ jets in events with the very low underlying multiplicities expected for these diffractive processes. It is likely that soft heavy quark production can be tagged through a secondary vertex at lower $p_{t}$. This would probe the lowest $x_{2}$ parton distributions(abscissa in Fig. 4)- possibly down to $x_{2} \sim 10^{-4}$. We also indicate in Fig. 4 the equivalent incident photon energy and the average rapidity $\left(y_{\text {jet }}\right)$ corresponding to each bin. For the most part, the produced jets are very central and it should be possible to require a large rapidity gap along the direction of the beam emitting the photon. The ATLAS forward calorimeters are unique in providing sensitivity up to $|\eta|<5.0$ for $E_{T} \geq 2.0 \mathrm{GeV}$ and this makes it the most promis- ing experiment to carry out rapidity gap physics with heavy ions.

\section{Acknowledgements}

I am grateful to the organizers of "Diffraction 2004" for a very stimulating and pleasant meeting.

\section{REFERENCES}

1. A. J. Baltz, C. Chasman and S. White, Nucl. Instr. And Meth. A417, 1 (1998).

2. C. Adler et al., Nucl. Instr. And Meth. A470, 488 (2001).

3. M. Chiu et al. Phys. Rev. Lett. 89 (2002) 012302, nucl-ex/0109018

4. H. Appelshauser et al.[NA49 Collaboration], Eur. Phys. J. A2, 383 (1998).

5. R. Snellings et al. Phys. Rev. Lett. 842803 (2000).

6. S. White "Applications of the Equivalent Photon Approximation to Heavy Ion Collisions" in "Electromagnetic Probes of Fundamental Physics" W. Marciano and S. White, eds. World Scinetific (2003).

7. G. Bauer et al. "Hot Topics in Ultraperipheral Heavy Ion Collisions", ibid.

8. E. Fermi (translation by M. Gallinaro and S. White) "On the Theory of Interactions between Atoms and Electrically Charged Particles", ibid.

9. G. Baur et al. Nucl.Phys.A729(2003) 787 and nucl-th/0307031

10. C. Adler et al. Phys.Rev.Lett. 89 (2002) 272302 and nucl-ex/0206004

11. A. J. Baltz, S. R. Klein, and J. Nystrand, Phys. Rev. Lett. 89012301 (2002).

12. For details see D. Silvermyr in www.phenix.bnl.gov/ silvermy/dnp04.pdf.

13. S. Klein and R. Vogt, Phys. Rev. C 68 (2003) 017902. and nucl-ex/0303013.

14. B. Kopeliovich, Phys. Rev. C 68 (2003) 044906 and nucl-th/0306044.

15. M. Strikman, R. Vogt and S. White manuscript in preparation. To be published in CERN yellow report on UltraPeripheral Collision physics and R. Vogt, hep-ph/0407298. 